\title{
Genomic sequencing and analysis of a Chinese hamster ovary cell line using Illumina sequencing technology
}

\author{
Stephanie Hammond ${ }^{1,2}$, Jeffrey C Swanberg ${ }^{1,2}$, Mihailo Kaplarevic ${ }^{2}$, Kelvin H Lee Le $^{1,2^{*}}$
}

\begin{abstract}
Background: Chinese hamster ovary $(\mathrm{CHO})$ cells are among the most widely used hosts for therapeutic protein production. Yet few genomic resources are available to aid in engineering high-producing cell lines.

Results: High-throughput Illumina sequencing was used to generate a 1x genomic coverage of an engineered $\mathrm{CHO}$ cell line expressing secreted alkaline phosphatase (SEAP). Reference-guided alignment and assembly produced 3.57 million contigs and CHO-specific sequence information for $\sim 18,000$ mouse and $\sim 19,000$ rat orthologous genes. The majority of these genes are involved in metabolic processes, cellular signaling, and transport and represent attractive targets for cell line engineering.
\end{abstract}

Conclusions: This demonstrates the applicability of next-generation sequencing technology and comparative genomic analysis in the development of $\mathrm{CHO}$ genomic resources.

\section{Background}

With over half of all recombinant therapeutic proteins produced in mammalian cell lines, Chinese hamster ovary $(\mathrm{CHO})$ cells remain the predominant production system for glycosylated biopharmaceuticals [1]. Although improvements in cell engineering, cell line selection, and culture conditions have increased productivity levels [2], the genetic basis underlying hyperproductivity remains poorly defined. The further development of genomic resources will facilitate detailed studies of genome structure, gene regulation, and gene expression in high-producing cell lines and aid in the use of sequence-specific molecular tools in cell line development.

A number of resources are required to support the assembly and annotation of the $\mathrm{CHO}$ genome including physical maps, genomic sequences, expressed sequence tag (EST) sequences, and proteomic data. Recent efforts to sequence and characterize bacterial artificial chromosome (BAC) libraries derived from $\mathrm{CHO}$ cells provide information for physical mapping of the $\mathrm{CHO}$ genome $[3,4]$. Transcriptomic and proteomic studies are

\footnotetext{
* Correspondence: KHL@udel.edu

'Department of Chemical Engineering, University of Delaware, Newark, DE 19711, USA

Full list of author information is available at the end of the article
}

currently used to examine differential expression of high-producing cell lines and to identify gene candidates for host cell engineering [5-7]. Transcriptomic studies which rely on cross-hybridization to mouse DNA microarrays showed some success [8,9], but also demonstrated the need for $\mathrm{CHO}$-specific sequence information. Continued EST sequencing of $\mathrm{CHO}$ cells lines has generated databases containing more than 60,000 sequences and allowed for the development of CHO-specific DNA microarrays $[10,11]$. Furthermore, mapping of CHO EST sequences to a mouse genomic scaffold can potentially reveal structural and regulatory features of the $\mathrm{CHO}$ genome [12]. Such studies are limited in that only a subset of genes expressed at sufficiently high levels are captured for sequence analysis, providing little information regarding genome structure or non-transcribed portions of the genome.

At present, there is little genomic sequence data available for $\mathrm{CHO}$ cells. This limits the application of highthroughput molecular tools in gene discovery and cell line engineering. $\mathrm{CHO}$ cell lines also undergo multiple genomic rearrangements during the generation of highproducing cell lines, necessitating the sequencing of individual cell lines rather than the Chinese hamster $[13,14]$. Until recently, EST sequences were obtained by
C Biomed Central

(c) 2011 Hammond et al; licensee BioMed Central Ltd. This is an Open Access article distributed under the terms of the Creative Commons Attribution License (http://creativecommons.org/licenses/by/2.0), which permits unrestricted use, distribution, and reproduction in any medium, provided the original work is properly cited. 
traditional Sanger technology [15], but current efforts are employing next-generation sequencing technologies including 454 and Illumina $[11,16,17]$. 454 pyrosequencing can generate up to $1 \mathrm{~Gb}$ of data in a single run, producing average read lengths of $330 \mathrm{bp}$ with an average error rate of $4 \%$, although a major limitation of this technology is the resolution of homopolymer regions $[18,19]$. Illumina sequencing can produce up to $90 \mathrm{~Gb}$ of data in a single run, generating reads up to $100 \mathrm{bp}$ in length with an average error rate of $1-1.5 \%[19,20]$. These technologies have significantly improved sequencing throughput and decreased cost, making mammalian genome sequencing feasible [20].

In this work, Illumina sequencing technology was used to generate an initial genomic sequence library of a Chinese hamster cell line with the goal of making these data available to the community. Comparative genomic analysis of this library was used to identify and functionally classify assembled sequences that were aligned to mouse and rat genes. An initial 1x coverage of the $\mathrm{CHO}$ cell genome provided $\mathrm{CHO}$-specific sequence information for a large number of protein coding genes, including those from functional classes typically underrepresented in EST libraries. This demonstrates that even low coverage genomic sequencing studies of $\mathrm{CHO}$ cell lines can increase the amount of sequence information available for this cell line.

\section{Results}

Illumina sequencing and reference-guided alignment Gene-amplified CHO-SEAP cells expressing human secreted alkaline phosphatase (SEAP) were previously generated from $\mathrm{CHO}$-DUK cells as a model for heterologous protein production [21]. Initial sequencing of the CHO-SEAP genome using Illumina technology yielded $2.72 \mathrm{~Gb}$ of genomic sequence, which represents $\mathrm{a} \sim 1 \mathrm{x}$ coverage of the $\mathrm{CHO}$ genome, estimated to be $2.8 \mathrm{~Gb}$ in size [4]. Reference-guided alignment was utilized in this study because a $1 \mathrm{x}$ genomic coverage is insufficient for de novo genome assembly. Previous work suggests that mouse and rat show a high degree of DNA sequence homology with the Chinese hamster [15] and several transcriptomic studies have employed a comparative approach to examine and annotate $\mathrm{CHO}$ sequence data $[12,16]$. Therefore, these species were chosen for comparative analysis. Reads were mapped to the reference genomes using MAQ software, which stands for Mapping and Assembling with Qualities [22]. Nearly 9\% of the total reads were aligned to both reference genomes, although a slightly higher number of reads were aligned to the mouse genome (Table 1). Each aligned read was assigned a mapping quality that indicates whether the read has a unique alignment or can be aligned to multiple positions in the genome [22]. Based on MAQ
Table 1 Alignment of $\mathrm{CHO}$ short reads to mouse and rat reference genomes

\begin{tabular}{ccccc}
\hline Sequence Type & \multicolumn{2}{c}{ Mouse } & \multicolumn{2}{c}{ Rat } \\
\hline Total aligned & $6,582,209$ & $(8.71 \%)$ & $6,281,589$ & $(8.31 \%)$ \\
Aligned to unique regions & $3,202,228$ & $(4.47 \%)$ & $3,046,308$ & $(4.28 \%)$ \\
Aligned to repeat regions & $3,379,981$ & $(4.24 \%)$ & $3,235,281$ & $(4.03 \%)$ \\
Aligned to protein-coding & $2,678,662$ & $(3.52 \%)$ & $2,359,457$ & $(3.12 \%)$ \\
genes* & & & & \\
\hline
\end{tabular}

* Pseudogenes, RNA genes, and genes on $Y$ chromosome were excluded from this analysis. Genes include exons, introns, and 5'- and 3'-untranslated regions

mapping qualities, $50 \%$ of the short reads from the raw data set were aligned to repetitive regions of the reference genomes (Table 1). In general, results from alignment to both mouse and rat reference genomes suggest that $\mathrm{CHO}$ genomic sequences are generally more similar to mouse genomic sequences, as previously demonstrated [15].

The distribution of CHO-SEAP reads aligned to the mouse and rat reference genomes was examined. The raw number of reads aligned was summed over $5 \mathrm{Mb}$ bins along reference chromosomes (Figure 1). Reads were mapped along all reference chromosomes, although several regions of higher coverage are present in both reference genomes. Many chromosomes in the current build of the mouse genome contain gaps in the 5 ' end of the reference sequence, which may account for the low number of reads mapping to the chromosome ends.

Simulation studies based on Sanger technology suggest that most of the genomic coding sequence can be surveyed with less than 2 -fold coverage [23] and low-coverage sequencing studies using Sanger [24] and nextgeneration $[25,26]$ sequencing technologies are successful in producing partial sequences of orthologous genes. Nearly $40 \%$ of the aligned CHO reads are mapped to protein-coding mouse and rat genes (Table 1). Sequence information was collected for $97 \%$ of known proteincoding genes in the mouse genome and 93\% of known protein-coding genes in the rat genome. To further examine gene coverage by this data set, the number of reads aligned to each gene in the mouse and rat protein-coding gene sets was examined (Figure 2). The raw read count was normalized by gene size to generate a normalized read count for each gene. This allows for a better comparison between the number of reads aligned to both very small, from thousands of bases, and very large, to millions of bases, genes. Only $3 \%$ of mouse genes and $7 \%$ of rat genes showed no coverage. Most genes showed low to medium coverage by the $\mathrm{CHO}$ short reads, with $33 \%$ of mouse and $48 \%$ of rat genes showing low coverage and $60 \%$ of mouse and $40 \%$ of rat genes showing medium coverage. A small proportion of both mouse and rat genes, less than $5 \%$, showed a high level of coverage. Because CHO-SEAP cells are 


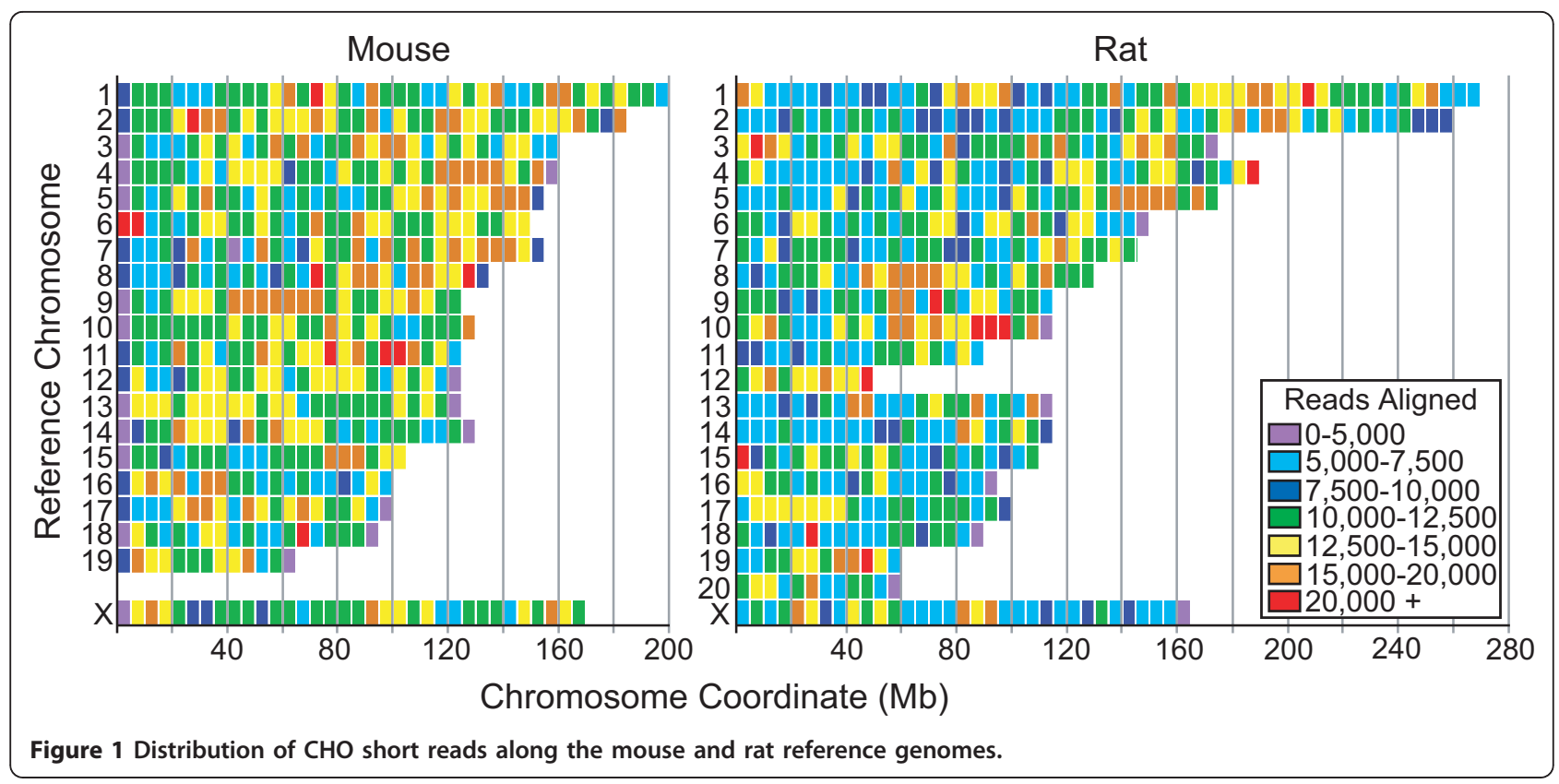

engineered to express high levels of a vector containing SEAP and dihydrofolate reductase (DHFR), the coverage of orthologous placental alkaline phosphatase and dihydrofolate reductase genes was examined. The placentallike alkaline phosphatase gene, Alppl2, showed medium to high coverage with 382 normalized read counts in rat

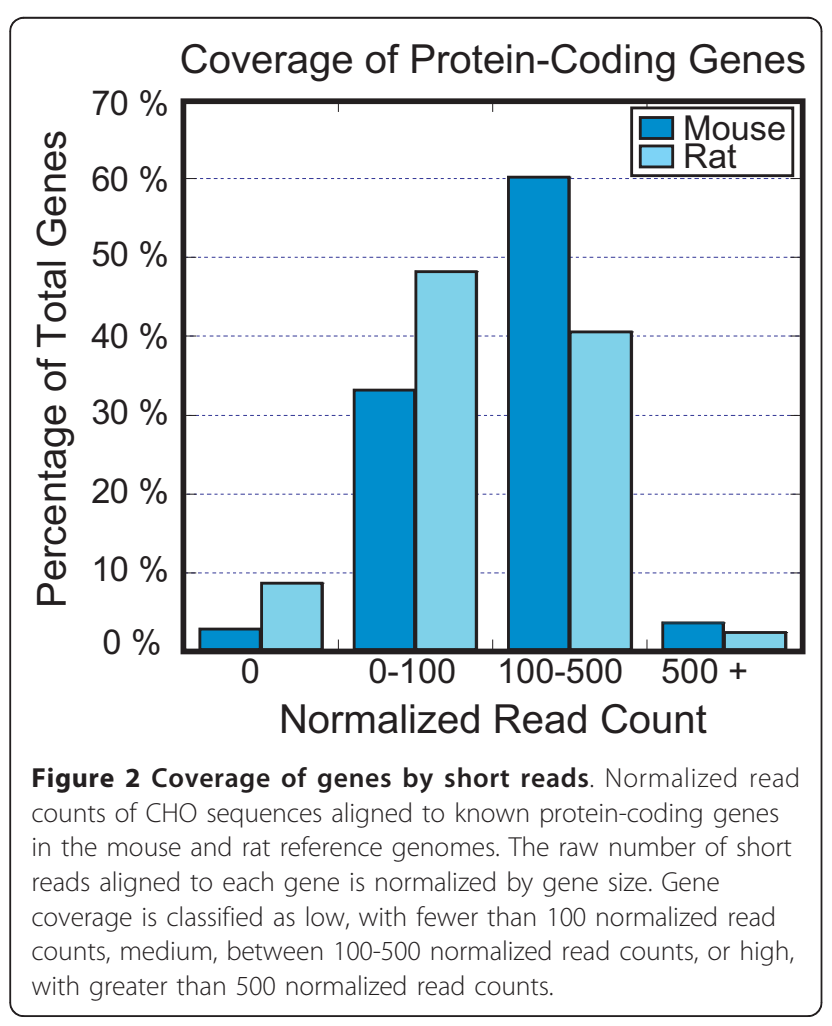

and 582 in mouse. The DHFR gene showed low coverage, with normalized read counts of 67 in mouse and 55 in rat. Genes with the highest coverage had 5,000 30,000 normalized read counts.

\section{Consensus sequence assembly and analysis}

To retrieve $\mathrm{CHO}$ sequences from this dataset, MAQ was used to assemble consensus genomic sequences of the mouse and rat alignments using sequence overlap information. In addition, performing the genomic assembly increased the length of the $\mathrm{CHO}$ sequences. Because of the 1-fold coverage of the $2.5 \mathrm{~Gb}$ mouse genome [27] and $2.75 \mathrm{~Gb}$ rat genome [28], a large proportion of the assembled sequences consisted of unsequenced bases, represented as N's in the consensus sequence. These files were parsed to extract $\mathrm{CHO}$ sequence contigs that range in size from 36 to $1,900 \mathrm{bp}$ and have an average length of $54 \mathrm{bp}$, representing a $50 \%$ increase in length over the 36 bp short reads. A total of 1.86 million contigs from alignment to the mouse genome and 1.71 million contigs from alignment to the rat genome were produced. The total combined length of $\mathrm{CHO}$ contigs from alignment to the mouse genome was 101.6 million bases and from alignment to the rat genome was 91.4 million bases. This corresponds to a $32-36 \%$ coverage of the $2.8 \mathrm{~Gb} \mathrm{CHO}$ genome. While $91 \%$ of these contigs are still short sequences less than $100 \mathrm{bp}$ in length, over 9\% exceed $100 \mathrm{bp}$ in length and a small fraction are larger than 500 bp (Table 2). The GC content of these contigs was analyzed. Rodent genomes show a higher average genomic GC content, $42 \%$ for mouse and slightly higher for rat, compared to an average genomic 
Table 2 Summary of CHO contigs extracted from consensus sequences

\begin{tabular}{|c|c|c|c|c|c|c|c|c|}
\hline \multirow{3}{*}{ Reference } & & & \multicolumn{6}{|c|}{ Contig Size } \\
\hline & \multicolumn{2}{|c|}{ Total } & \multicolumn{2}{|c|}{$<100 \mathrm{bp}$} & \multicolumn{2}{|c|}{$\begin{array}{c}100 \text { to } 500 \\
\text { bp }\end{array}$} & \multicolumn{2}{|c|}{$>500 \mathrm{bp}$} \\
\hline & $\begin{array}{l}\text { Size } \\
(b p)\end{array}$ & $\begin{array}{l}\% \\
\mathrm{GC}\end{array}$ & $\begin{array}{l}\text { Size } \\
\text { (bp) }\end{array}$ & $\begin{array}{l}\% \\
\text { GC }\end{array}$ & $\begin{array}{l}\text { Size } \\
(b p)\end{array}$ & $\begin{array}{l}\% \\
\text { GC }\end{array}$ & $\begin{array}{l}\text { Size } \\
(b p)\end{array}$ & $\begin{array}{l}\% \\
\mathrm{GC}\end{array}$ \\
\hline Mouse & 54 & 43.0 & 46 & 42.9 & 140 & 44.0 & 637 & 45.2 \\
\hline Rat & 53 & 42.9 & 46 & 42.8 & 139 & 44.2 & 616 & 46.5 \\
\hline
\end{tabular}

The average size and average GC content of $\mathrm{CHO}$ contigs generated from reference-guided alignment

GC content of $41 \%$ for human [27,28]. Overall, $\mathrm{CHO}$ contigs generated from alignment to the mouse and rat genomes show an average GC content of $43.0 \%$ and $42.9 \%$, respectively. The GC content increases as contig length increases (Table 2).

This assembly set was annotated by comparing $\mathrm{CHO}$ contigs against custom genomic databases using a basic local alignment search tool (BLAST) algorithm [29]. Approximately $30 \%$ of these contigs resulted in significant matches in the genomic databases (Table 3). The high average similarity of these hits, 96\%, was expected due to the stringent alignment criteria, including the low number of allowed mismatches. Contigs that resulted in BLAST hits ranged in size from 36 to $1,000 \mathrm{bp}$, with an average size of $60 \mathrm{bp}$. A total of 563,163 contigs resulted in BLAST hits to non-protein coding regions of the genome, providing sequence information that is unavailable from EST sequencing experiments. An additional 479,270 contigs hit known protein-coding mouse and rat genes, providing $\mathrm{CHO}$ specific sequence information for 17,883 mouse and 19,481 rat genes.

$\mathrm{CHO}$ contigs were examined in the context of the reference genomic structures. Regions within the consensus sequences were aligned to genes of interest in the mouse genome using BLAT [30] at the UCSC genome browser [31]. CHO contigs aligned to DHFR, the amplification marker, and placental alkaline phosphatase, the recombinant protein produced in this cell line, were chosen because of the interest in these genes for cell line development efforts. Contigs mapped within the coding regions of the mouse DHFR and Alppl2 genes

Table 3 Summary of assembly and BLAST analysis of CHO sequences

\begin{tabular}{ccc}
\hline & Mouse & Rat \\
\hline Total contigs & $1,864,122$ & $1,707,312$ \\
Contigs with BLAST hits & 559,545 & 482,888 \\
Average\% similarity of BLAST hits & $96.46 \%$ & $96.39 \%$ \\
Contigs hit known genes & 264,917 & 214,353 \\
Total unique genes hit & 17,883 & 19,481 \\
\hline
\end{tabular}

are shown (Figure 3A-B). Two contigs of average size, 49 and 67 bp in length, separated by a small stretch of unsequenced bases mapped to an exon in Alppl2 (Figure 3C) and closer examination of this alignment reveals several nucleotide differences in the $\mathrm{CHO}$ contigs relative to the mouse reference sequence (Figure 3D).

Gene ontology (GO) analysis was used to examine the functional classes of genes for which $\mathrm{CHO}$ sequences had been generated. GO terms were retrieved for the 17,883 mouse and 19,481 rat genes identified in the BLAST analysis of $\mathrm{CHO}$ contigs. A similar distribution of functional gene classes was observed for both mouse and rat assembly data sets (Figure 4). The top three functional groups identified in this analysis are genes associated with metabolism $(\sim 36 \%)$, intracellular and extracellular signaling $(\sim 30 \%)$ and transport ( 17\%). Although genes related to metabolic processes and vesicle-mediated transport are highly represented in EST sequencing libraries, genes involved in cellular signaling pathways are poorly represented [11]. Therefore, genomic sequencing of $\mathrm{CHO}$ cell lines provides additional sequence information on members of this and other functional classes that may be underrepresented in current EST libraries.

\section{Discussion}

A comparative genomics approach was used to generate sequence-specific information for a high-producing $\mathrm{CHO}$ cell line with the goal of making this data publicly available. The development of $\mathrm{CHO}$ genomic resources will benefit not only cell line engineering efforts to enhance biopharmaceutical production but other areas of research utilizing $\mathrm{CHO}$ cells, such as the use of radiation hybrid mapping for comparative genomic analysis [32]. The analysis presented here demonstrates the potential of applying Illumina sequencing in the development of CHO genomic resources. Integration of genomic sequences derived from multiple next-generation technologies, such as 454 and Illumina sequencing, with those derived from Sanger sequencing enhance genomic coverage [33]. The inclusion of long paired-end or mate-paired libraries, with varied insert sizes, coupled with the high-throughput of next-generation sequencing technologies should also provide not only sequence but structural information required for de novo assembly of the $\mathrm{CHO}$ genome.

Neither the short reads nor the reference genomes were repeat-masked prior to alignment. A prevalent feature of mammalian genomes is the high content of repetitive sequences. Approximately $46 \%$ of the human genome, $37 \%$ of the mouse genome, and $40 \%$ of the rat genome are repetitive sequences $[27,28]$. Repeat-masking either the short reads or reference genome would discard information about a significant fraction of the 


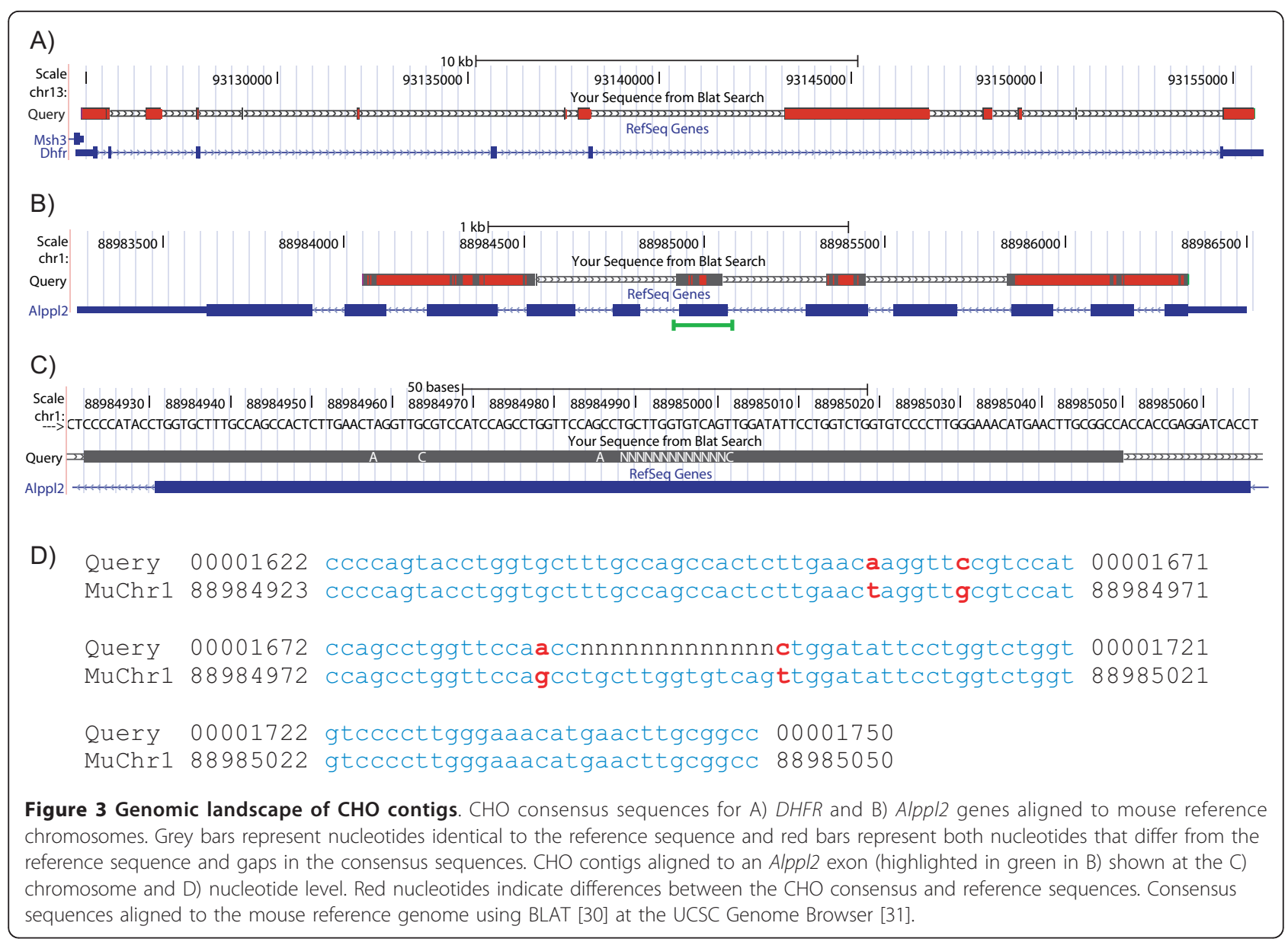

genome and would reduce coverage in an uneven manner [22]. Recent work suggests that endogenous repetitive structures on $\mathrm{CHO}$ chromosomes may promote gene amplification and increase the stability of the amplified gene [3]. Including repetitive regions in the assembly and analysis may help identify genomic structures associated with hyperproductive $\mathrm{CHO}$ cell lines.

Several studies employed a similar approach to successfully generate genomic resources for non-model organisms from low-coverage data $[25,34,35]$. There are

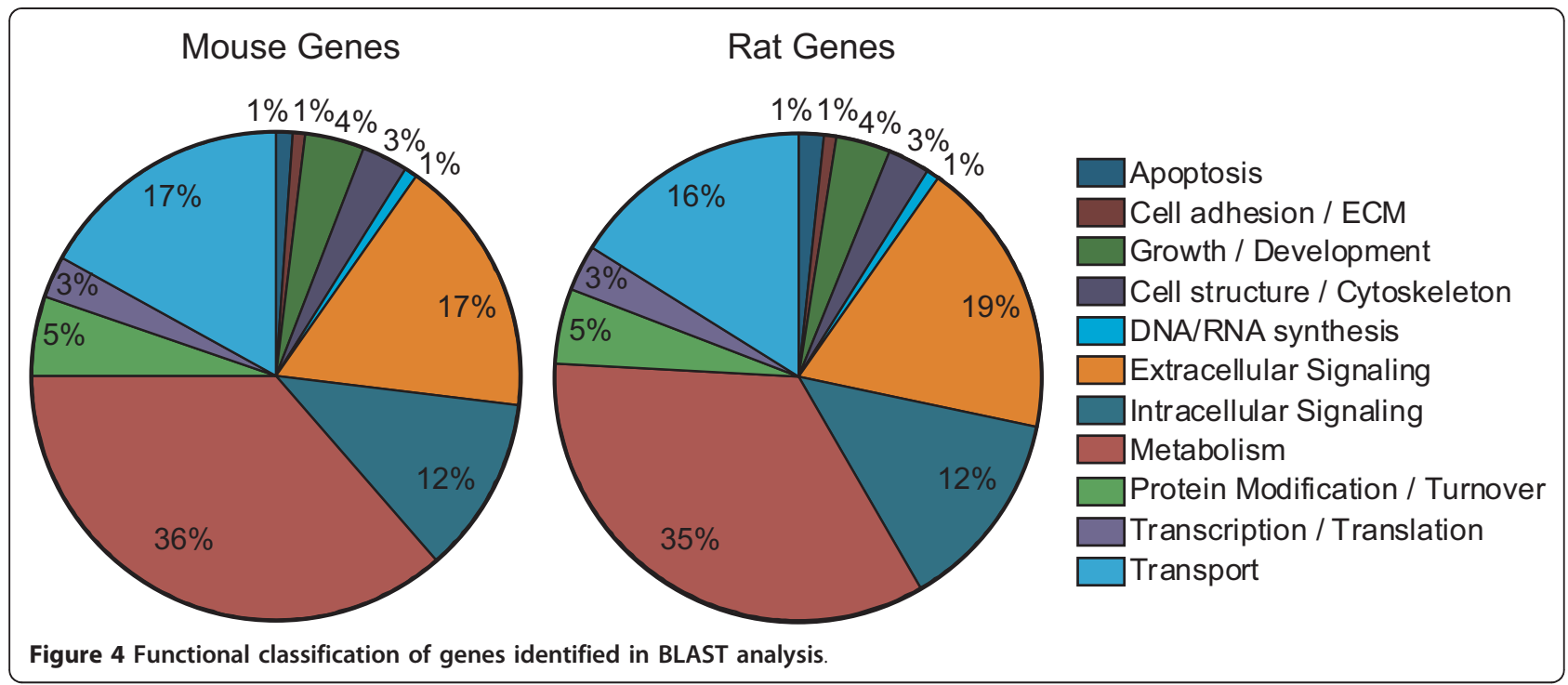


inherently some limitations to a reference-guided alignment and analysis regarding sequence similarity and genomic structure. MAQ allows up to 2 mismatches within the first $28 \mathrm{bp}$ of each read and does not allow for gaps in the alignment [22]. Short reads derived from regions with less than $94 \%$ identity to the reference sequence may not be aligned [36]. This may account for the low percentage of total $\mathrm{CHO}$ reads aligned to either the mouse or rat genome and suggests that the $\mathrm{CHO}$ contigs presented here represent highly conserved regions between $\mathrm{CHO}$ cells and mouse or rat. In an initial genomic sequencing of the turkey using Illumina technology, only one-third of the short 35 bp reads could be directly aligned to the chicken genome, a closely related species, suggesting that a large portion of the short reads may not be expected to align in this type of analysis [34].

Additionally, during alignment, the sequenced genome is scaffolded onto the reference, so the structure of the final consensus sequence may not be representative of the true genomic architecture [36]. New methodologies are being developed to improve the consensus genomic sequences produced by reference-guided alignment $[37,38]$. CHO cell lines commonly used in biopharmaceutical production have a reduced chromosome number compared to primary Chinese hamster cells [4]. These cell lines also undergo genomic rearrangements as a result of amplification procedures used to develop high-producing cell lines $[13,14]$. Therefore, the genomic structure of the Chinese hamster may not be representative of the individual cell lines and analysis of specific $\mathrm{CHO}$ cell lines may provide a better understanding of the structural changes associated with hyperproductivity.

Of particular interest in $\mathrm{CHO}$ cell lines is examining the relationship between the location of the amplified gene and productivity of the cell line. BAC libraries were recently used to examine the site and structure of the transgene vector in gene-amplified cell lines [3,4]. The DHFR amplicon is large, up to several hundreds of thousands of nucleotides, and may contain repeated segments of the endogenous $\mathrm{CHO}$ genome $[3,4,39]$. The small lengths of the $\mathrm{CHO}$ contigs makes it unlikely that any contig will span both the DHFR amplicon and the host genome. Additionally, the transgene vector sequence is not present in the reference genome used during alignment. This makes it difficult to determine the integration site of the DHFR transgene vector in this analysis. A greater coverage of the $\mathrm{CHO}$ genome to permit de novo assembly of the reads will facilitate determining the integration site and copy number of the DHFR amplicon in this cell line. Increased coverage and refinement of the $\mathrm{CHO}$ genome will also enable detection of other copy number variants, such as insertions and deletions, and accurate SNP identification to assist cell line engineering efforts [40-42].

\section{Conclusions}

The complexity of the $\mathrm{CHO}$ genome, including the structural rearrangements that occur during gene amplification and cell line derivation, makes assembly of a genomic sequence challenging. Next-generation sequencing technologies allow for the rapid acquisition of genomic sequence from $\mathrm{CHO}$ cell lines. This sequencing information can be used to generate a draft genome sequence when coupled with physical maps that can be derived from BAC libraries and a $\mathrm{CHO}$ scaffold that can be derived from cross-species comparative analysis. Incorporation of additional sequence data from transcriptomic studies and EST libraries will be necessary for complete annotation. The development of these resources is required to fully utilize sequence-specific tools, such as DNA microarrays and RNA interference, in cell line development and to understand how gene regulation and genome structure is altered in high-producing cell lines.

\section{Methods}

\section{Genomic library construction and Illumina sequencing}

$\mathrm{CHO}$ cells engineered to express human secreted alkaline phosphatase (SEAP) were generated from CHO-DUK cells (ATCC 9096) as described previously [21]. CHOSEAP cells were maintained as adherent cultures in IMDM (Iscove's modified Dulbecco's medium, Invitrogen, Carlsbad, CA) supplemented with 10\% dFBS (dialyzed fetal bovine serum, Invitrogen) and $5120 \mathrm{nM}$ methotrexate (Calbiochem, San Diego, CA). Genomic DNA from CHO-SEAP cells was isolated using the Genomic DNA mini kit (Invitrogen). A single-end library was prepared using the DNA sample kit (Illumina, San Diego, CA) according to manufacturer's instructions. The genomic library was sequenced on an Illumina GA system at the Cornell University Life Sciences Core Laboratory Center (Ithaca, NY) by running 36 cycles according to manufacturer's instructions. Approximately $2.72 \mathrm{~Gb}$ from $75,583,814$ high quality reads passed the Illumina GA Pipeline filter and were used for alignment. FASTQ files containing raw sequences and sequence qualities were deposited at the National Center for Biotechnology Information Sequence Read Archive (NCBI SRA) under the accession SRA012218. While analysis of SRA data sets is computationally challenging, rapid improvements in assembly algorithms and computational power are enabling more researchers to benefit from this type of data set.

\section{Sequence alignment and assembly}

Reference genomes for mouse chromosomes 1-19 and X (M_musculus Build 37) and rat chromosomes 1-20 and 
X (R_norvegicus Build 3.4) were obtained from the NCBI genomic download site [43]. Reference guided alignment to both mouse and rat reference genomes and consensus sequence assembly was performed with MAQ 0.7.1 [22] using default settings. MapView was used for visual inspection of alignments [44]. Aligned reads were analyzed to determine if they mapped to unique or repetitive genomic regions, based on mapping qualities, or within protein-coding genes, based on genomic coordinates, using MATLAB (The MathWorks, Inc., Natick, MA). To verify sequencing reliability, the short read data set was aligned to the Chinese hamster mitochondrial genome (NC_007936.1) and resulted in significant homology.

\section{Gene coverage of protein-coding gene sets}

Known protein-coding gene sets for both mouse and rat were established as follows: genomic coordinates for mouse and rat genes were retrieved from Mouse Genome Informatics (MGI) [45] and the Rat Genome Database (RGD) [46] and filtered to retain only known protein-coding genes. The mouse protein-coding gene sets contains 21,691 genes from chromosomes 1-19 and $\mathrm{X}$ and the rat protein-coding gene set contains 26,450 genes from chromosomes 1-20 and X. Gene size is defined from the genomic coordinates from MGI and RGD and includes exons, introns, and untranslated regions.

Normalized read counts were calculated for each gene in the protein-coding gene sets to which short reads were mapped. A normalization factor was calculated by dividing the size of each gene by the average gene size in the protein-coding gene sets, with an average gene size of 44,862 bp for mouse and 34,186 bp for rat. Normalized read counts were determined by dividing the raw number of reads aligned to each gene by the normalization factor calculated for that gene.

\section{Functional analysis of genomic assembly}

Gene names and Gene Ontology (GO) terms were assigned to all contigs that shared sequence similarity with known protein-coding mouse and rat genes. Contigs were extracted from consensus sequences using Python and custom scripts. Contigs were aligned to reference genomes using BLAT [30] and viewed using the UCSC Genome Browser [31]. Sequence comparisons were done using standalone BLAST from NCBI [29]. Custom genomic databases were generated from mouse and rat reference chromosomes. Contigs were mapped to these genomic databases using BLASTN with a significance threshold of $\mathrm{e}<1^{-10}$. BLAST outputs were parsed using Perl scripts to retrieve the best hit for each contig. Gene names and GO terms were retrieved for each contig that hit a known protein-coding gene. GO IDs for mouse (NCBIM37) and rat (RGSC3.4) genes were retrieved from ENSEMBL (release 56) using BioMart [47]. GO analysis was performed using the CateGOrizer web tool [48].

\section{Authors' contributions}

SH performed data analysis and drafted the manuscript, JCS prepared samples and participated in data analysis, MK performed data analysis, KHL conceived the project. All authors have read and approved the final manuscript. The corresponding author will gladly provide detailed information on the cluster hardware setup, analysis software version and parameters, upon request.

\section{Acknowledgements}

This work was supported by Cornell University and the University of Delaware. The authors thank Peter Schweitzer at the Cornell Biotechnology Resource Center for Illumina sequencing and Manoj Pillay for assistance in processing consensus sequences.

\section{Author details}

1Department of Chemical Engineering, University of Delaware, Newark, DE 19711, USA. ²Delaware Biotechnology Institute, University of Delaware, Newark, DE 19711, USA.

Received: 14 September 2010 Accepted: 26 January 2011

Published: 26 January 2011

\section{References}

1. Wurm FM: Production of recombinant protein therapeutics in cultivated mammalian cells. Nat Biotechnol 2004, 22:1393-1398.

2. Kuystermans D, Krampe B, Swiderek H, Al-Rubeai M: Using cell engineering and omic tools for the improvement of cell culture processes. Cytotechnology 2007, 53:3-22.

3. Park JY, Takagi Y, Yamatani M, Honda K, Asakawa S, Shimizu N, Omasa T, Ohtake $\mathrm{H}$ : Identification and analysis of specific chromosomal region adjacent to exogenous Dhfr-amplified region in Chinese hamster ovary cell genome. J Biosci Bioeng 2010, 109:504-511.

4. Omasa T, Cao Y, Park JY, Takagi Y, Kimura S, Yano H, Honda K, Asakawa S, Shimizu N, Ohtake H: Bacterial artificial chromosome library for genomewide analysis of Chinese hamster ovary cells. Biotechnol Bioeng 2009, 104:986-994.

5. Pascoe DE, Arnott D, Papoutsakis ET, Miller WM, Andersen DC: Proteome analysis of antibody-producing $\mathrm{CHO}$ cell lines with different metabolic profiles. Biotechnol Bioeng 2007, 98:391-410.

6. Nissom PM, Sanny A, Kok YJ, Hiang YT, Chuah SH, Shing TK, Lee YY, Wong KT, Hu WS, Sim MY, Philp R: Transcriptome and proteome profiling to understanding the biology of high productivity $\mathrm{CHO}$ cells. $\mathrm{MO}$ Biotechnol 2006, 34:125-140.

7. Yee JC, Gerdtzen ZP, Hu WS: Comparative transcriptome analysis to unveil genes affecting recombinant protein productivity in mammalian cells. Biotechnol Bioeng 2009, 102:246-263.

8. Ernst W, Trummer $\mathrm{E}$, Mead J, Bessant $\mathrm{C}$, Strelec $\mathrm{H}$, Katinger $\mathrm{H}$, Hesse $\mathrm{F}$ : Evaluation of a genomics platform for cross-species transcriptome analysis of recombinant CHO cells. Biotechnol J 2006, 1:639-650.

9. Yee JC, Wlaschin KF, Chuah SH, Nissom PM, Hu WS: Quality assessment of cross-species hybridization of $\mathrm{CHO}$ transcriptome on a mouse DNA oligo microarray. Biotechnol Bioeng 2008, 101:1359-1365.

10. Bahr SM, Borgschulte T, Kayser KJ, Lin N: Using microarray technology to select housekeeping genes in Chinese hamster ovary cells. Biotechnol Bioeng 2009, 104:1041-1046.

11. Kantardjieff A, Nissom PM, Chuah SH, Yusufi F, Jacob NM, Mulukutla BC, Yap M, Hu WS: Developing genomic platforms for Chinese hamster ovary cells. Biotechnol Adv 2009, 27:1028-1035.

12. Wlaschin KF, Hu WS: A scaffold for the Chinese hamster genome. Biotechnol Bioeng 2007, 98:429-439. 
13. Derouazi M, Martinet D, Besuchet Schmutz N, Flaction R, Wicht M, Bertschinger M, Hacker DL, Beckmann JS, Wurm FM: Genetic characterization of $\mathrm{CHO}$ production host DG44 and derivative recombinant cell lines. Biochem Biophys Res Commun 2006, 340:1069-1077.

14. Ruiz JC, Wahl GM: Chromosomal destabilization during gene amplification. Mol Cell Biol 1990, 10:3056-3066.

15. Wlaschin KF, Nissom PM, Gatti Mde L, Ong PF, Arleen S, Tan KS, Rink A, Cham B, Wong K, Yap M, Hu WS: EST sequencing for gene discovery in Chinese hamster ovary cells. Biotechnol Bioeng 2005, 91:592-606.

16. Birzele F, Schaub J, Rust W, Clemens C, Baum P, Kaufmann H, Weith A, Schulz TW, Hildebrandt T: Into the unknown: expression profiling without genome sequence information in $\mathrm{CHO}$ by next generation sequencing. Nucleic Acids Res 2010, 38:3999-4010.

17. Jacob NM, Kantardjieff A, Yusufi FN, Retzel EF, Mulukutla BC, Chuah SH, Yap M, Hu WS: Reaching the depth of the Chinese hamster ovary cell transcriptome. Biotechnol Bioeng 2010, 105:1002-1009.

18. Margulies M, Egholm M, Altman WE, Attiya S, Bader JS, Bemben LA, Berka J, Braverman MS, Chen YJ, Chen Z, et al: Genome sequencing in microfabricated high-density picolitre reactors. Nature 2005, 437:376-380

19. Shendure J, Ji H: Next-generation DNA sequencing. Nat Biotechnol 2008, 26:1135-1145

20. Metzker ML: Sequencing technologies - the next generation. Nat Rev Genet 2010, 11:31-46.

21. Hayduk EJ, Lee KH: Cytochalasin D can improve heterologous protein productivity in adherent Chinese hamster ovary cells. Biotechnol Bioeng 2005, 90:354-364.

22. Li H, Ruan J, Durbin R: Mapping short DNA sequencing reads and calling variants using mapping quality scores. Genome Res 2008, 18:1851-1858.

23. Bouck J, Miller W, Gorrell JH, Muzny D, Gibbs RA: Analysis of the quality and utility of random shotgun sequencing at low redundancies. Genome Res 1998, 8:1074-1084.

24. Kirkness EF, Bafna V, Halpern AL, Levy S, Remington K, Rusch DB, Delcher AL, Pop M, Wang W, Fraser CM, Venter JC: The dog genome: survey sequencing and comparative analysis. Science 2003, 301:1898-1903.

25. Rasmussen DA, Noor MA: What can you do with $0.1 \times$ genome coverage? A case study based on a genome survey of the scuttle fly Megaselia scalaris (Phoridae). BMC Genomics 2009, 10:382.

26. Bai $X$, Zhang $W$, Orantes $L$, Jun TH, Mittapalli $O$, Mian MA, Michel AP: Combining next-generation sequencing strategies for rapid molecular resource development from an invasive aphid species, Aphis glycines. PLoS One 2010, 5:e11370.

27. Waterston $\mathrm{RH}$, Lindblad-Toh K, Birney E, Rogers J, Abril JF, Agarwal P, Agarwala R, Ainscough R, Alexandersson M, An P, et al: Initial sequencing and comparative analysis of the mouse genome. Nature 2002, 420:520-562.

28. Gibbs RA, Weinstock GM, Metzker ML, Muzny DM, Sodergren EJ, Scherer S, Scott G, Steffen D, Worley KC, Burch PE, et al: Genome sequence of the Brown Norway rat yields insights into mammalian evolution. Nature 2004, 428:493-521.

29. Camacho C, Coulouris G, Avagyan V, Ma N, Papadopoulos J, Bealer K, Madden TL: BLAST+: architecture and applications. BMC Bioinformatics 2009, 10:421.

30. Kent WJ: BLAT-the BLAST-like alignment tool. Genome Res 2002, 12:656-664.

31. The UCSC Genome Browser. [http://genome.ucsc.edu]

32. Murphy WJ, Stanyon R, O'Brien SJ: Evolution of mammalian genome organization inferred from comparative gene mapping. Genome Biol 2001, 2, REVIEWSO005.

33. Quinn NL, Levenkova N, Chow W, Bouffard P, Boroevich KA, Knight JR, Jarvie TP, Lubieniecki KP, Desany BA, Koop BF, et al: Assessing the feasibility of GS FLX Pyrosequencing for sequencing the Atlantic salmon genome. BMC Genomics 2008, 9:404.

34. Kerstens HH, Crooijmans RP, Veenendaal A, Dibbits BW, Chin AWTF, den Dunnen JT, Groenen MA: Large scale single nucleotide polymorphism discovery in unsequenced genomes using second generation high throughput sequencing technology: applied to turkey. BMC Genomics 2009, 10:479.

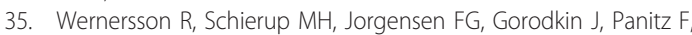
Staerfeldt $\mathrm{HH}$, Christensen OF, Mailund T, Hornshoj $H$, Klein A, et al: Pigs in sequence space: a $0.66 \mathrm{X}$ coverage pig genome survey based on shotgun sequencing. BMC Genomics 2005, 6:70

36. Dutilh BE, Huynen MA, Strous M: Increasing the coverage of a metapopulation consensus genome by iterative read mapping and assembly. Bioinformatics 2009, 25:2878-2881.

37. Schneeberger K, Hagmann J, Ossowski S, Warthmann N, Gesing S, Kohlbacher $\mathrm{O}$, Weigel D: Simultaneous alignment of short reads against multiple genomes. Genome Biol 2009, 10:R98.

38. Gnerre S, Lander ES, Lindblad-Toh K, Jaffe DB: Assisted assembly: how to improve a de novo genome assembly by using related species. Genome Biol 2009, 10:R88.

39. Wurm FM, Petropoulos CJ: Plasmid integration, amplification and cytogenetics in CHO cells: questions and comments. Biologicals 1994, 22:95-102

40. Yoon S, Xuan Z, Makarov V, Ye K, Sebat J: Sensitive and accurate detection of copy number variants using read depth of coverage. Genome Res 2009, 19:1586-1592.

41. Medvedev P, Stanciu M, Brudno M: Computational methods for discovering structural variation with next-generation sequencing. Nat Methods 2009, 6:S13-20.

42. Liao PY, Lee KH: From SNPs to functional polymorphism: The insight into biotechnology applications. Biochem Eng J 2010, 49:149-158.

43. NCBI Genomes FTP site. [ftp://ftp.ncbi.nih.gov/genomes/].

44. Bao H, Guo H, Wang J, Zhou R, Lu X, Shi S: MapView: visualization of short reads alignment on a desktop computer. Bioinformatics 2009, 25:1554-1555.

45. Mouse Genome Informatics. [http://www.informatics.jax.org]

46. Rat Genome Database. [http://rgd.mcw.edu/]

47. BioMart. [http://www.biomart.org].

48. Zhi-Liang H, Bao J, Reecy JM: CateGOrizer: A Web-Based Program to Batch Analyze Gene Ontology Classification Categories. Online J Bioinformatics 2008, 9:108-112.

doi:10.1186/1471-2164-12-67

Cite this article as: Hammond et al:: Genomic sequencing and analysis of a Chinese hamster ovary cell line using Illumina sequencing technology. BMC Genomics 2011 12:67.

\section{Submit your next manuscript to BioMed Central and take full advantage of:}

- Convenient online submission

- Thorough peer review

- No space constraints or color figure charges

- Immediate publication on acceptance

- Inclusion in PubMed, CAS, Scopus and Google Scholar

- Research which is freely available for redistribution

Submit your manuscript at www.biomedcentral.com/submit
C) Biomed Central 